\title{
ESTADO DE CONOCIMIENTO DE LOS TECAMEBIANOS DULCEACUICOLAS DE CHILE
}

\author{
CURRENT STATE OF KNOWLEDGE OF FRESHWATER \\ THECAMOEBIANS OF CHILE
}

\author{
Jaime Zapata Madrid \\ Universidad de Los Lagos. Depto. Ciencias Básicas. Casilla 933, Osorno. \\ Email: jzapata@ulagos.cl
}

\begin{abstract}
RESUMEN
Las tecamebas son un grupo de protozoos que viven principalmente en las comunidades acuáticas y de ambientes húmedos (suelos, turberas, etc.), en donde juegan un importante rol como descomponedoras de celulosa y lignina; además participan activamente en diversos procesos biológicos que ocurren en los ecosistemas acuáticos. Con respecto al conocimiento de las tecamebas en Chile, éste se mantiene en un estado muy incipiente, de ahí la necesidad de contar con más especialistas, ya que este grupo está adquiriendo cada vez más importancia por su uso en problemas de paleolimnología, como bioindicadores de ambientes lacustres contaminados, como indicadores pedológicos, entre otros
\end{abstract}

Palabras Claves: Protozoa, tecamebas, taxonomía, Chile.

\begin{abstract}
The Thecamoebians is a protozoa group that live mainly in aquatic communities and environments with sufficient moisture (soils, peat bogs, etc.); since they take an active part as primary destructors of cellulose and lignin and they also participate actively in diverse biological processes that happen in the water ecosystems. The knowledge of the Thecamoebians in Chile stays in a very incipient state creating the necessity to count with more specialist, since this group is acquiring more and more importance for its use in understanding paleolimnological problems, as bio-indicators of polluted lacustrine environments, as pedological indicators, etc.
\end{abstract}

KEYwords: Protozoa, thecamoebians, taxonomy, Chile.

\section{INTRODUCCION}

Los tecamebianos o testáceos (también citados como "arceláceos", "rizópodos aglutinados" y "amebas tecadas") son organismos unicelulares $(60-300 \mu \mathrm{m})$ provistos de una teca o conchilla rígida y de seudópodos de naturaleza variable. El nombre de estos protozoarios es informal, ya que corresponde a un grupo polifilético artificial que incluye parte de la Clase Rhizopodea (Subclase Lobosia, Orden Arcellinida) y parte de la Clase Reticularea (Subclase
Filosia, Orden Gromida; y probablemente parte del Suborden Allogromiina) (Loeblich \& Tappan 1964). La teca puede ser secretada por el propio organismo (teca autógena) y su naturaleza puede ser proteínica, silícea y raramente calcárea. Pero, la mayoría de las especies tienen su teca formada de partículas extrañas (xenosomas) aglutinadas con cemento autógeno (teca xenógena). Este cemento es un ácido mucopolisacárido con calcio, hierro, manganeso, fósforo y trazas de cloro y potasio (Ogden 1979, 1988); y los xenosomas pueden ser granos de arena $y /$ 
o frústulas de diatomeas. Se las encuentra en agua dulce (ríos y lagos), agua salobre, en el suelo, humus, turberas, tubo digestivo de batracios, de lombrices, en musgos, líquenes, hojas de árboles, etc. (Medioli \& Scott 1988); además, sirven de alimento a hidrobiontes más grandes, como: ciliados libres, oligoquetos, nemátodos, larvas y pequeños peces (Alekperov \& Snegovaya 1999). Su registro fósil, especialmente de las aglutinadas, data del Carbonífero $(\sim 250 \mathrm{~m} . \mathrm{a})$, aunque su mayor abundancia ocurre en el PleistocenoHoloceno (Wightman et al. 1994).

\section{TRABAJOS PRECEDENTES}

El conocimiento de las tecamebas de Chile se inició con Ehrenberg (1843), quien estudió material del Cabo de Hornos y señaló a Difflugia phiale, Difflugia ermitana y Difflugia antarctica (la que corresponde a alguna Euglypha). Posteriormente, Certes (1891) analizó sedimento recolectado en Bahía Orange (Tierra del Fuego) por la Misión del Cabo de Hornos (18821883) desde riachuelos que llegaban a la bahía, logrando determinar 24 especies de rizópodos testáceos. El estudio de estos organismos prosiguió con Wailes (1913) al reconocer seis especies en charcas de Antofagasta y siete en Valparaíso, y en charcas, arroyos y musgos de Punta Arenas determinó 36 especies. A este trabajo le siguió el de Jung (1942), autor que analizó muestras recolectadas en las Termas de Puyehue, Isla Calbuco, Río Palena, Lago Risopatrón, Puerto Puyuhuapi, Isla Puluqui, etc. En este trabajo se mencionaron 158 especies, incluyéndose en esta cifra especies ya conocidas, y especies, variedades, formas y combinaciones nuevas. Del estudio realizado por Hoogenraad \& Groot (1951) de musgos conservados en herbarios provenientes de Brasil y Chile, se entregó un listado de 48 especies de tecamebas. La importancia pedológica de estos organismos fue manifestada por Bonnet (1966) en su análisis microfaunístico de algunos suelos de Chile (Cordillera Pelada: La Unión; Vega del Flaco: Cordillera de San Fernando), de lo cuales logró identificar 94 especies y variedades de tecamebianos. Algo más reciente es el trabajo de Zapata \& Rudolph (1986) acerca de las tecamebas bentónicas, planctónicas y epífitas del Río Damas (Osorno); de su listado de 39 especies, ellos describieron y figuraron 13 especies como nuevas para Chile. Seguidamente, Zapata \& Matamala (1987) tuvieron a su disposición material recolectado en el Lago Kitiesh (Antártica), del cual identificaron 15 especies. En el trabajo de Zapata \& Crespo (1990) se describieron y figuraron 12 especies de tecamebas encontradas en sedimento proveniente de una laguna del volcán Rano-Kau (Isla de Pascua). Finalmente, Zapata et al. (2002) estudiaron la distribución de las tecamebas en el estuario del Río Contaco (Osorno), logrando determinar cuatro familias, seis géneros y 21 especies; además, concluyeron que la salinidad era el factor predominante en la distribución de las especies a lo largo del río.

\section{TAXONOMÍA}

Para la identificación de las especies de tecamebas se usan principalmente: la forma y composición de la teca, forma de su abertura, presencia de espinas, de un cuello, etc. Pero, las variaciones morfológicas infraespecíficas (morfotipos) producidas por la influencia de ciertos factores ambientales deben ser tomadas en cuenta al delimitar las especies. Así, algunos investigadores han considerado a cada variante como una especie nueva, mientras que otros consideran a cada variante como perteneciente a la misma especie (Medioli \& Scott 1983, McCarthy 1984). Por lo tanto, la taxonomía de este grupo está aún poco clara, debido a los problemas nomenclaturales y taxonómicos, ya que muchos taxa han sido descritos superficialmente y basados casi enteramente en la morfología de la teca (Medioli \& Scott 1985, Foissner \& Korganova 1995). Sin embargo, recientes estudios recomiendan apoyarse también con suficientes datos morfométricos, puesto que numerosas subespecies, variedades y formas existentes caen en el rango de la variabilidad natural de las especies (Wanner 1991, Foissner \& Korganova 1995).

\section{UTILIDAD PRÁCTICA DEL GRUPO}

Las tecamebas tienen una generación en pocos días y la descendencia puede desarrollar diferente morfología de acuerdo a las variaciones de las condiciones ambientales. Estas variaciones infraespecíficas ("strains") han sido utilizadas con éxito para distinguir subambientes no reconocibles al usar las especies. Algunos usos actuales son: 1) Como indicadoras de ambientes lacustres contaminados por residuos químicos de minas e industrias (Asioli et al. 1996, Patterson et al. 1996, Reinhardt et al. 1998), ya que algunas especies son extremadamente resistentes a 
los ambientes contaminados; a la vez que otras especies son muy sensibles a los cambios naturales y a aquellos producidos por el hombre en ambientes lacustres, tales como el pH, la contaminación por metales pesados, etc. (Collins et al. 1990, Patterson et al. 1997, Kumar \& Patterson 2000, Patterson \& Kumar 2000). 2) Su uso para determinar el grado de eutrofia de lagos, debido a que muchas especies prefieren lagos oligotróficos con leve acidez y un reducido número de especies prefieren lagos eutróficos (Medioli et al. 1990). 3) Para establecer los cambios paleoambientales y paleoclimáticos ocurridos en ambientes lacustres durante el Pleistoceno-Holoceno (Medioli \& Scott 1988, McCarthy et al. 1995). 4). Como indicadoras pedológicas, debido a la especificidad que tienen por determinados biotopos terrestres, motivo por el cual son consideradas como los protozoos más importantes del suelo por su gran biomasa y producción (Foissner 1987, 1999). 5) Para conocer la respuesta de los microorganismos a la acción de la luz ultravioleta (Searles et al. 2001).

\section{Situación ACtuAl del CONOCimiento EN Chile}

En Chile actualmente existe un sólo investigador, en la Universidad de Los Lagos-Osorno, que estudia a las tecamebas desde el punto de vista sistemático y en menor grado zoogeográfico. Tal situación deja un campo muy extenso para el estudio de estos organismos, en áreas como la zoogeografía, taxonomía, paleolimnología, como bioindicadores de contaminación en lagos y ríos, como indicadores paleoecológicos, distribución en ambientes mixohalinos de estuarios y lagunas costeras, plancton $\mathrm{y}$ bentos de lagunas y lagos andinos, turberas, pantanos, etc. Además se hace necesaria implementar una colección de referencia de tecamebas chilenas para los investigadores interesados en el grupo, la cual podría ubicarse en una determinada universidad.

\section{BIBLIOGRAFIA}

Alekperov, I. \& N. Snegovaya. 1999. Specific composition and number of Testaceous Amoebae (Testacea Lobosia, Protozoa) of Ganli-Gol Lake. Journal of Zoology 23: 313-319.

Asioli, A., F. Medioli \& R. Patterson. 1996. Thecamoebians as a tool for reconstruction of paleoenvironments in some Italian lakes in the foothills of the southern Alps (Orta, Varese and Candia). Journal of Foraminiferal Research 26(3): 248-263.

Bonnet, L. 1966. Le peuplement thécamoebien de quelques sols du Chili. Protistologica 2(2): 113140.

Certes, A. 1891. Protozoaires. Mission Scientiphique du Cap Horn (1882-1883), Zoologie, 6: 1-53.

Collins, E., F. McCarthy, F. Medioli, D. Scott \& D. Honig. 1990. Biogeographic distribution of modern thecamoebians in a transect along the eastern North American coast. NATO Advanced Study Inst. Series C, Mathematics and Physics 327: 783-791.

Ehrenberg, C. 1843. Verbreitung und Einfluss des mikroskopischen Lebens in Süd-und Nord Amerika. Königliche Akad. der Wissenschaften zur Berlin (1841): p. 291-446.

FoISSNER, W. 1987. Soil protozoa: fundamental problems, ecological significance, adaptations in ciliates and testaceans, bioindicators, and guide to the literature. Protistology 2: 69-212.

FoISSNER, W. 1999. Soil protozoa as bioindicators: pros and cons, methods, diversity, representative examples. Agriculture, Ecosystems and Environment 74: 95-112.

FoIssner, W. \& G. Korganova. 1995. Redescription of three testate amoebae (Protozoa, Rhizopoda) from Caucasian soil: Centropyxis plagiostoma Bonnet \& Thomas, Cyclopyxis kahli (Deflandre) and $C$. intermedia Kufferath. Archiv für Protistenkunde 146: 13-28.

HoogenraAd, H. \& A. Groot. 1951. Thekamoebe Mossrhizopoden aus Südamerika. Archiv für Hydrobiologie 45: 346-366.

Jung, W. 1942. Südchilenische Thekamöben. Archiv für Protistenkunde 95: 253-356.

Kumar, A. \& R. Patterson. 2000. Arcellaceans (Thecamoebians): New tools for monitoring long and short term changes in lake bottom acidity. Environmental Geology 39(6): 689-697.

Loeblich, A. \& H. Tappan. 1964. Sarcodina, chiefly "Thecamoebians" and Foraminiferida. En: Treatise on Invertebrate Paleontology (Ed. R. Moore), pp. 1-510. Geological Society of America and University of Kansas.

McCARTHY, F. 1984. Infraspecific variation in Arcellacea and a selected biostratigraphic study. Unpublished B.Sc. Honours Thesis, Dalhousie University.

McCarthy, F., E. Collins, J. McAndrews, H. Kerr, D. Scott \& F. Medioli. 1995. A comparison of postglacial Arcellacean (Thecamoebians) and pollen succession in Atlantic Canada, illustrating the potential of arcellaceans for paleoclimatic reconstruction. Journal of Paleontology 69(5): 980-993.

Medioli, F. \& D. Scott. 1983. Holocene Arcellacea (Thecamoebians) from eastern Canada. Cushman Foundation for Foraminiferal Research 21: 5-63.

Medioli, F. \& D. Scott. 1985. Designation of types, for 
Gayana 70(1), 2006

one genus and nine species of Arcellaceans (Thecamoebians) with additional reference material for four other species. Journal of Foraminiferal Research 15: 24-37.

Medioli, F. \& D. Scott. 1988. Lacustrine thecamoebians (mainly Arcellaceans) as potential tools for paleolimnological interpretations. Paleogeography, Paleoclimatology, Paleoecology 62: 361-386.

Medioli, F., Scott, D., Collins, E. \& F. McCarthy. 1990. Fossil thecamoebians: present status and prospects for the future. En: Paleoecology, Biostratigraphy, Paleoceanography and Taxonomy of Agglutinated Foraminifera (Eds. C. Hemleben, M. Kaminski, W. Kuhnt \& D. Scott), pp. 813-840. NATO Advanced Study Institute Series C, Mathematics and Physics.

Ogden, C. 1979. Comparative morphology of some pyriform species of Difflugia (Rhizopoda). Archiv für Protistenkunde 122: 143-153.

OGDEN, C. 1988. Morphology of the organic shell matrix of Difflugia (Rhizopoda) in culture, including modifications by the addition of agglutinate particles. Archiv für Protistenkunde 136: 365-376.

Patterson, R., T. Barker \& S. Burbidge. 1996. Arcellaceans (Thecamoebians) as proxies of arsenic and mercury contamination in northeastern Ontario Lakes. Journal of Foraminiferal Research 26: 172-183.

Patterson, R., A. Kumar \& C. Dumaresq. 1997. Arcellaceans: shelled microinvertebrates as costeffective tools for monitoring current and historical environmental trends. $24^{\text {th }}$ Annual Aquatic Toxicity Workshop, Niagara Falls, Ontario, :19-22.

Patterson, R. \& A. Kumar. 2000. Assessment of Arcellaceans (Thecamoebians) assemblages, species, and strains as contaminant indicators in James Lake, northeastern Ontario, Canada. Journal of Foraminiferal Research 30(4): 310-320.

Reinhardt, E., A. Dalby, A. Kumar \& R. Patterson. 1998. Arcellaceans as pollution indicators in mine tailing contaminated lakes near Cobalt, Ontario, Canada. Micropaleontology 44: 131-148.

Searles, P., B. Kropp, S. Flint \& M. Caldwell. 2001. Influence of solar UV-B radiation on peatland microbial communities of southern Argentina. New Phytologist 152: 213-221.

Wailes, G. 1913. Freshwater Rhizopods from North and South America. Journal of Linnean Society, Zoology 32: 121-161.

Wanner, M. 1991. Morphologie von Thekamöben (Protozoa: Rhizopoda) in süddeutschen Wäldern. Archiv für Protistenkunde 140: 45-66.

Wightman, W., D. Scott, F. Medioli \& M. Gibling. 1994. Agglutinated foraminifera and thecamoebians from the Late Carboniferous Sydney coal field, Nova Scotia: Paleoecology, paleoenvironments and paleogeographical implications. Paleogeography, Paleoclimatology, Paleoecology 106: 187202.

Zapata, J. \& E. RudolPh. 1986. Tecamebas del Río Damas $\left(40^{\circ} 34^{\prime} \mathrm{S} ; 73^{\circ} 08^{\prime} \mathrm{W}\right)$, Chile. Biota, Chile, 2 : 6582.

Zapata, J. \& M. Matamala. 1987. Tecamebianos del Lago Kitiesh (Isla Rey Jorge, Antártica). Biota, Chile, 3: 59-71.

ZAPATA, J. \& J. CRESPO. 1990. Tecamebas del volcán RanoKau, Isla de Pascua (2710’ S; $109^{\circ} 26^{\prime}$ W). Biota, Chile, 6: 53-59.

Zapata, J., P. Alvarez \& C. Cea. 2002. Tecamebas del Río Contaco (40³3’12” S; 7343’00” W), Osorno, Chile. Boletín de la Sociedad de Biología de Concepción, Chile, 73: 17-35.

Fecha de recepción: 03.08.05

Fecha de aceptación: 22.11.05 